\title{
Réponse dynamique des systèmes d'exploitation offshore soumis à la houle et au courant en grande profondeur d'eau
}

\author{
Dynamic behaviour of offshore floating production systems in deep water
}

\author{
par C. Berhault et G. Morin \\ PRINCIPIA R.
}

After a short description of offshore systems proposed for oil and gas production in ultra deep water the first part of paper presents the physical aspects of their dynamic behaviour. The second part is devoted to the classical numerical tools used for dynamic analysis of floating vessels and for the risers analysis. As these tools have some drastic limitations, the main research activities are addressed to unsolved problems. Two of them are discussed in the paper: the vortex induced vibrations of risers and the wave non linearities and impacts on the vessels.

\section{ARCHITECTURE DES SYSTÈMES DE FORAGE EN GRANDE PROFONDEUR}

Les gisements découverts récemment par des profondeurs d'eau supérieures à 1000 mètres et dans différentes zones géographiques ont conduit les opérateurs pétroliers à rechercher de nouvelles solutions technologiques pour les exploiter. Ces solutions sont basées sur l'utilisation de supports flottants, d'architectures variées suivant leurs fonctions (forage, production, stockage) et de liaisons de grandes longueurs reliant le fond et la surface (Fig. 1). Du point de vue du dimensionnement des structures soumises à la houle et au courant, forer ou exploiter en grande profondeur d'eau pose alors deux problèmes majeurs : le choix du système d'ancrage des supports flottants et le comportement des liaisons fond / surface.

\subsection{Ancrage des supports flottants}

Le premier problème est le maintien sur site du support flottant. Le recours aux lignes d'ancrage traditionnelles (chaine) n'est plus possible pour des raisons de poids et d'encombrement. On utilise alors des lignes composites constituées essentiellement de câble acier et/ou de matériaux synthétiques. Une alternative est le positionnement dynamique, utilisé pour le forage et avec des limitations d'utilisation. Afin de réduire les déports statiques sous les efforts de vent, de courant et de houle, les lignes d'ancrage sont relativement tendues. Elles sont donc très sollicitées dynamiquement par les mouvements du support flottant et il n'est en général plus possible de découpler le mouvement du support de la réponse des lignes. Les sollicitations dues à l'environnement peuvent être décrites de la manière suivante (Fig.2) [1] :

- la houle, le vent et le courant engendrent des efforts moyens qui tendent à déporter le support flottant. Ces efforts sont contrés par le rappel des lignes d'ancrage,

- les efforts de houle sur le flotteur engendrent deux types de réponse dynamique : une composante aux fréquences de houle qui sollicite dynamiquement l'ancrage et une composante aux modes propres du système flotteur/ancrage, de fréquences basses devant celles de la houle. Cette dernière composante est particulièrement non-linéaire,

- les efforts de traînée sur les lignes d'ancrage, induits par

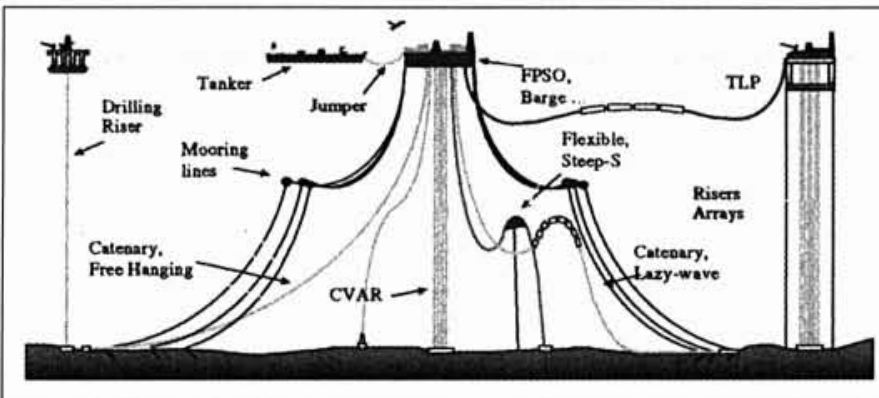

1. Architecture pour le forage et la production offshore. 


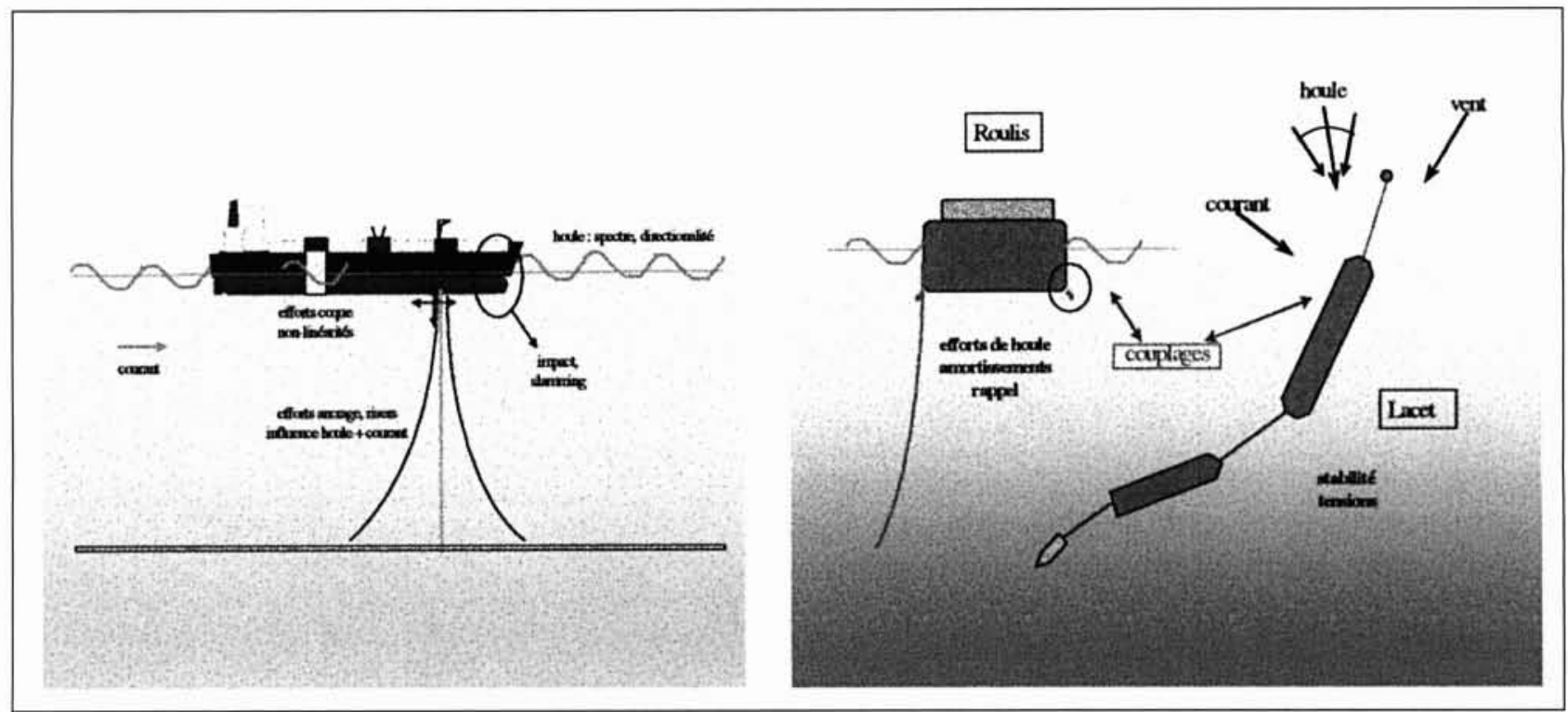

2-a. Sollicitation dynamique d'un support flottant.

l'écoulement relatif local, contribuent significativement à l'amortissement de la composante résonante,

- dans les houles très sévères, les efforts d'impact de la houle sur la coque, le slamming et l'embarquement d'eau sur le pont engendrent des efforts transitoires supplémentaires.

\subsection{Comportement des conduites de forage et de production (risers)}

Le problème le plus important concerne le dimensionnement des conduites de forage et de production. Ces conduites, de section circulaire, ont des diamètres extérieurs variant de quelques dizaines de centimètres à 2,0 mètres. La structure d'une section de conduite est généralement complexe pour assurer les différentes fonctions : flexibilité, isolation ther- mique, résistance aux pressions interne et externe. Le diamètre externe n'est pas nécessairement constant avec la profondeur d'eau. Des conduites de diamètres différents peuvent également interférer. Les architectures d'ensemble proposées sont très variées : conduite de forage suspendue au navire et encastrée au fond ou librement suspendu, riser rigide caténaire ou flexible, riser auto-porteur. Le comportement dynamique d'un riser est principalement gouverné par (Fig.2) [8] :

- les efforts de traînée dus au courant qui induisent une déflection moyenne. Le courant peut affecter toute la tranche d'eau avec des variations en intensité et en direction,

- les efforts de traînée et d'inertie induits par le champ de vagues et par les mouvements du navire dans la partie supérieure de la tranche d'eau. Ces efforts engendrent une réponse dynamique aux fréquences de la houle incidente,

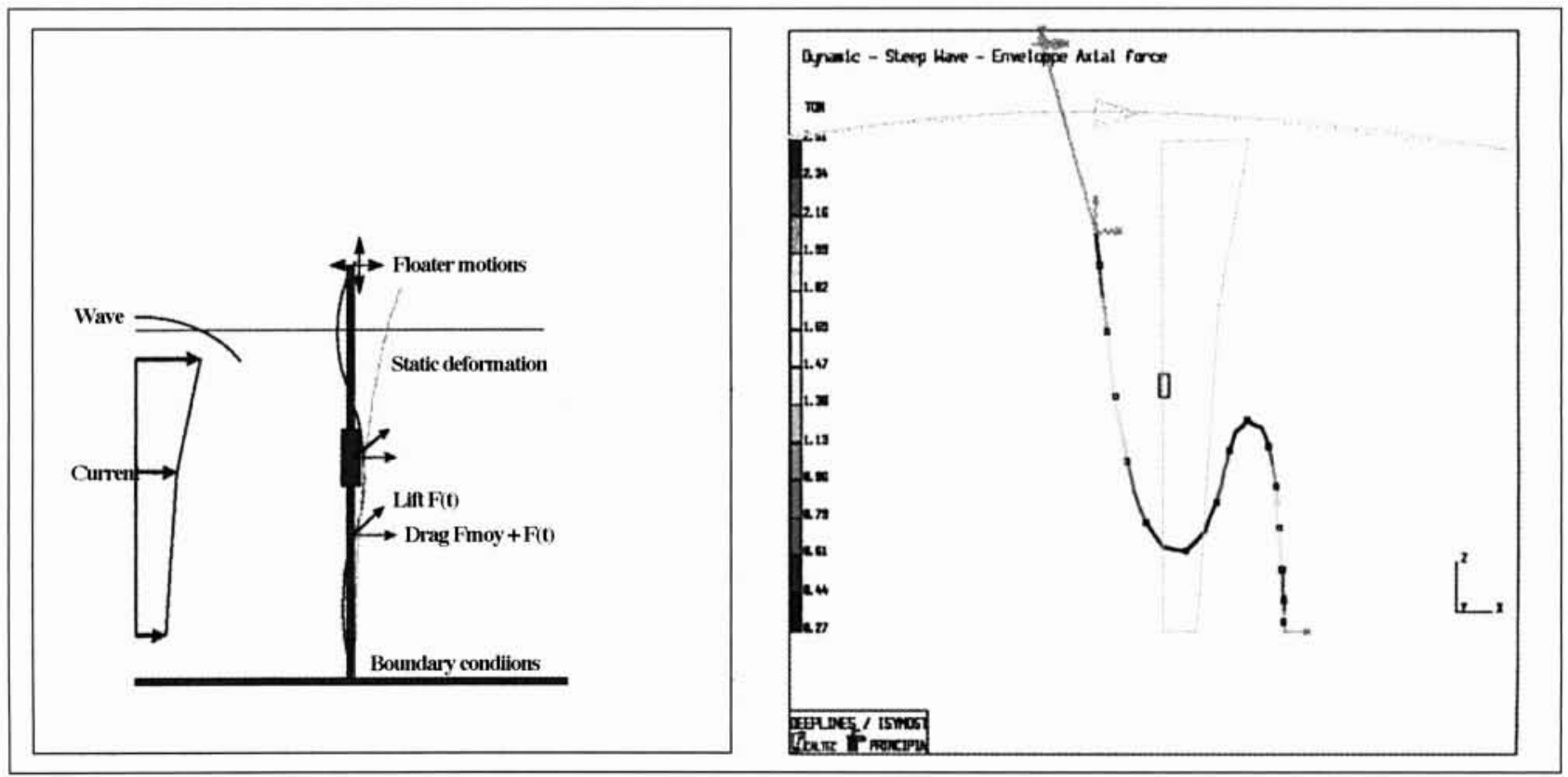

2-b. Sollicitation dynamique d'une conduite de production - Modèle éléments finis. 
- les efforts de portance et de traînée induits par les détachements tourbillonnaires alternés qui engendrent des vibrations transverses aux modes propres haute fréquence de la conduite (VIV). Une variation de courant sur la tranche d'eau peut exciter simultanément plusieurs modes transverses et axiaux du riser.

Les VIV contribuent largement à la fatigue du riser tout en accroissant les efforts de traînée dans la direction du courant. Une attention particulière est donc apportée à leur prédiction.

\section{II — MÉTHODES D'ANALYSE ET OUTILS DE CALCUL ACTUELS}

\section{- 2.1 Réponse dynamique des supports flottants}

Les outils de calcul du comportement des supports ancrés soumis à la houle, au courant et au vent sont maintenant largement utilisés dans les projets industriels. Ils sont basés sur les hypothèses et les méthodes suivantes :

- les efforts de houle sont calculés en théorie potentielle (écoulement irrotationnel de fluide parfait). La cambrure de la houle et les mouvements du flotteur sont supposés petits pour linéariser les conditions aux limites. Les problèmes dits de diffraction et de radiation sont résolus en domaine fréquentiel jusqu'au $2^{\text {ème }}$ ordre d'approximation. La méthode de résolution la plus utilisée a recours à une formulation en intégrales de frontière et fait appel à une fonction de Green satisfaisant la condition de surface libre linéarisée. Sa mise en œuvre nécessite une discrétisation de la partie immergée du flotteur, voire d'une partie de la surface libre si le couplage avec le courant est introduit. Les fonctions de transfert des efforts sont déduites. Les interactions hydrodynamiques entre plusieurs flotteurs peuvent êtres prises en compte.

- après linéarisation, les équations du mouvement du flotteur permettent d'accéder à la réponse aux fréquences de houle et aux pressions dynamiques sur la carène. Ces pressions, ajoutées aux pressions hydrostatiques, sont projetées sur un maillage en éléments finis du flotteur pour réaliser, à l'aide d'un logiciel de calcul de structure, la vérification du dimensionnement de la coque (efforts globaux, contraintes locales) pour différents états de mer. A ce stade, la réaction de l'ancrage n'a que peu d'influence. Par contre, il se peut qu'une fréquence propre du flotteur corresponde à la gamme des fréquences de houle. Dans ce cas, l'approche théorique utilisée n'est plus licite. Il faut en particulier prendre en compte la traînée visqueuse sur la carène qui agit comme amortissement. L'approche est alors le plus souvent empirique : essais en oscillations libres ou forcées et identification des efforts d'amortissement à une formulation analytique. L'utilisation de fonctions de transfert permet d'évaluer les spectres de réponse dès lors que le spectre de houle est connu. Les valeurs extrêmes et les écarts-type en sont déduits moyennant des hypothèses sur les lois de distribution des pics. - les efforts dans les ancrages sont estimés à partir de l'intégration en temps des équations du système dynamique flotteurs / lignes d'ancrage [1]. Cette approche permet de prendre en compte, d'une part, les différentes composantes fréquentielles des efforts de houle (fréquences de houle et $2^{\text {nd }}$ ordre basse fréquence) et, d'autre part, les différentes non-linéarités qui interviennent dans les efforts de réaction (hydrostatique, ancrage) et dans les efforts d'amortissement (traînée sur la carène et sur les lignes d'ancrage). Les efforts de courant et de vent sur les différentes composantes du système sont inclus. Les modèles les plus élaborés sont conçus pour traiter des configurations multi-structures avec différents types de liaison et d'événements, y compris transitoires (contacts, vérinage, ballastage, ruptures) [2].

Les outils de calcul actuels traitent donc de manière découplée la réponse dynamique des structures et l'écoulement fluide. Ce découplage est réalisé en imposant des hypothèses (fluide parfait, faible déplacement des structures) qui peuvent apparaître inconsistantes avec l'utilisation des résultats pour déterminer les réponses extrêmes des structures. Les modèles de réponse dynamique sont alors complétés avec des termes plus ou moins empiriques, issus de l'expérience, qui introduisent des couplages : formulation des efforts de traînée en vitesse relative fluide / structure, correction des efforts de houle pour les vagues très cambrées : c'est la "cuisine" de l'hydrodynamicien. Cette approche présente l'inconvénient d'être difficilement généralisable. Des adaptations sont nécessaires pour chaque type d'architecture. Par contre son avantage est d'être aisée et rapide d'utilisation : l'analyse et l'optimisation d'un système sont réalisées en quelques jours sur PC.

\subsection{Réponse dynamique des liaisons fond / surface}

La réponse statique et dynamique des conduites reliant le fond de la mer à la surface est obtenue par des modèles de type éléments finis adaptés aux structures filaires. Compte tenu de la complexité structurelle d'une conduite, ces modèles ont été étendus pour prendre en compte les réactions axiales, en flexion, en torsion, les singularités mécaniques (flotteurs, connecteurs, tensionneurs, flex-joint), les interférences entre plusieurs conduites, les contacts, le frottement sur le sol, l'écoulement interne [8].

La résolution s'effectue soit dans le domaine fréquentiel, après linéarisation, soit dans le domaine temporel. Plus récemment les équations du mouvement des supports flottants ont été introduites dans ces modèles pour obtenir la réponse dynamique couplée entre flotteurs et liaisons fond / surface (Fig.3).

Les efforts hydrodynamiques sont obtenus à partir de formulations de type Morison avec là encore une "cuisine hydrodynamique" plus ou moins sophistiquée basée sur des données expérimentales. L'hypothèse principale est que l'écoulement local est bidimensionnel et gouverné par la vitesse relative entre l'écoulement incident (courant, houle) et le déplacement de la structure. Il faut alors fournir les coefficients d'inertie et de traînée correspondants. Ces coefficients sont censés représenter toute la complexité de

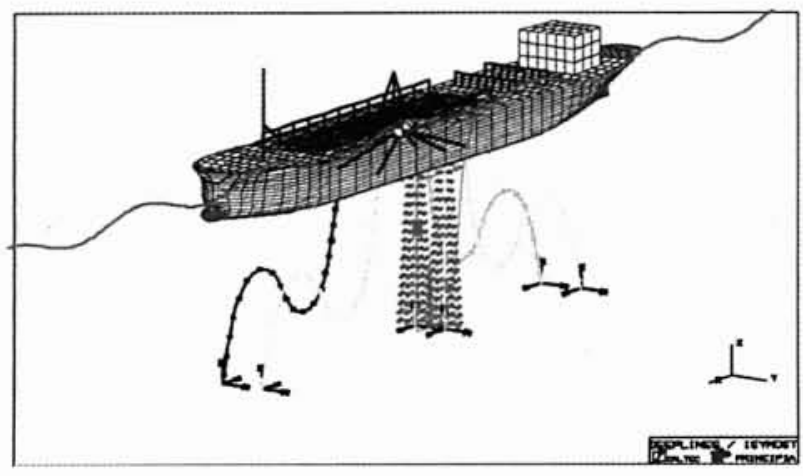

3. Couplage support de surface / liaison fond / surface. 
l'écoulement : géométrie de la section, dépendance des nombres de Reynolds et de Keulegan et Carpenter, interférences entre risers. Le caractère tridimensionnel de la réponse du riser est introduit en imposant des efforts de type Morison dans les deux directions transverses et dans la direction axiale avec des coefficients hydrodynamiques différents. Cette approche présente deux inconvénients pour l'étude des configurations en grande profondeur d'eau :

- la réponse dynamique de la conduite contient une large gamme de modes de vibration autour de sa déformée moyenne : fréquences de la houle, fréquences des mouvements du flotteur de surface, fréquences de lock-in.

- les vibrations in-line, transverses et axiales sont couplées du fait du comportement 3D de la conduite. L'écoulement devient lui-même 3D, accentuant le couplage entre les modes.

Il devient alors difficile de définir les paramètres de références utilisés pour choisir les coefficients hydrodynamiques. Le plus souvent il faut avoir recours à l'analyse découplée des différentes composantes fréquentielles, puis imposer les coefficients pour chaque composante.

Un problème particulier est celui des vibrations induites par les vortex qui contribuent à la fatigue quand la fréquence de Strouhal locale s'accorde avec celle d'un mode propre de la structure (lock-in). Les modèles existants, développés pour la vibration des câbles, supposent un courant constant et font l'hypothèse de la superposition des modes excités sans prendre en compte les amortissements possibles dans leur propagation, ce qui conduit à une surestimation de la fatigue. Les efforts hydrodynamiques sont encore une fois déduits de formulations empiriques de type Morison introduisant une composante transverse fluctuante. Les longueurs de corrélation associées à l'évolution des points de décollement le long de la conduite ne sont connues que pour des cylindres rigides. Pour des cylindres mobiles elles sont largement influencées par les amplitudes et les fréquences des vibrations. Les vibrations transverses accroissent également la traînée moyenne in-line. Dès que le courant est variable en intensité et en direction dans la tranche d'eau ou dès que la conduite est soumise à des mouvements d'extrémité (support de surface) plusieurs modes peuvent être excités simultanément à différents niveaux et dans différentes directions. Ces modes se propagent en se combinant. Les singularités géométriques rencontrées (variation de diamètre, flotteurs) contribuent à l'amortissement de même que les interférences hydrodynamiques entre les conduites voisines. Il apparaît alors, par comparaison avec les essais, que les modèles existants sont peu fiables.

En conclusion, les ingénieurs disposent de modèles numériques très élaborés pour prédire le comportement dynamique des conduites pétrolières, hormis les vibrations induites par les tourbillons. Ces modèles nécessitent cependant une bonne expertise pour le choix des coefficients hydrodynamiques. Le recours à des données expérimentales est le plus souvent nécessaire, données qui, en général, sont conservées jalousement.

\section{III - LES ACTIONS DE R\&D ET LA NOU- VELLE GÉNÉRATION DE MODÈLES}

\section{- 3.1 Tendances}

Avant de présenter les deux principaux thèmes de recherche concernant le comportement dynamique des structures offshore, il paraît intéressant de préciser les orienta- tions générales poursuivies pour disposer d'une nouvelle génération de modèles numériques :

- modéliser les déformées exactes de surface libre en présence d'une structure : fortes cambrures, déferlement, runup, franchissement [3]. Les développements sont poursuivis en $2 \mathrm{D}$ et $3 \mathrm{D}$ aussi bien en hypothèse de fluide parfait (équations intégrales, [10]) qu'en fluide visqueux [7], voire par le couplage des deux approches [9]. Les difficultés sont dans la restitution de la houle incidente et dans les simulations de longue durée :

- résoudre simultanément les équations du mouvement de la structure avec les équations de l'écoulement. Pour une structure rigide, la difficulté est dans le suivi de l'intersection carène-surface libre afin d'obtenir une évaluation précise des efforts hydrostatiques [7]. Pour une structure déformable, deux approches sont poursuivies. La première consiste à projeter la déformée structurelle (coque, cylindre flexible) sur la base de ses modes propres, puis à les introduire dans le modèle de calcul d'écoulement. La déformée structurelle est alors obtenue à chaque instant, puis prise en compte pour le calcul des pressions et des vitesses fluide. La deuxième approche consiste à résoudre simultanément les équations de l'écoulement fluide et celles de la structure sans hypothèses restrictives. Les tentatives actuelles consistent à réaliser un couplage entre un logiciel de calcul de structure non-linéaire et un logiciel de résolution des équations de Navier-Stokes ;

- modéliser correctement les sillages en écoulement instationnaire complexe : écoulement oscillant multi-fréquentiel, interactions entre plusieurs structures mobiles.

\subsection{Vibrations induites par les tourbillons}

Le problème des vibrations induites par les tourbillons (VIV) est l'objet d'un effort important de recherche aussi bien au niveau français qu'international. L'objectif final est de disposer d'outils de calcul couplant les logiciels éléments finis avec la résolution des équations de Navier-Stokes. Dans le projet coopératif Hydlines mené depuis 1998 dans le cadre du Clarom, la démarche suivante a été adoptée :

- réalisation d'essais $2 \mathrm{D}$ pour un cylindre rigide monté sur appuis élastiques et tracté dans un bassin de grande longueur (canal de traction de l'Ecole Centrale de Nantes) à des nombres de Reynolds voisins de $10^{5}$. Il existe en effet de nombreuses données pour les Reynolds correspondant au régime sous critique (zone à faible variation du Strouhal). Par contre elles sont peu nombreuses pour les Reynolds correspondant à la zone de transition, zone de grand intérêt pour les risers. Si dans cette zone le sillage devient désordonné pour un cylindre fixe, dans le cas d'un cylindre mobile une réorganisation peut être observée par effet de lock-in. L'apparition des vibrations peut être influencée par les conditions initiales et des comportements transitoires. Les VIV deviennent alors de nature chaotique.

- adaptations et validation en écoulement 2D instationnaire de modèles de résolution des équations de Navier-Stokes incluant les équations du mouvement du cylindre. Un travail de recherche important est consacré à la modélisation de la turbulence et la modélisation des interférences entre plusieurs cylindres [5]. Pour ce faire, le projet dispose également de résultats d'essais sur la vibration de plusieurs cylindres sur appuis élastiques à faible nombre de Reynolds. - réalisation d'essais 3D à faible nombre de Reynolds pour 
un cylindre flexible tendu dans un bassin avec courant (BGO First). L'objectif est d'analyser la composition de plusieurs modes de vibration (Strouhal) engendrés à différents niveaux du flexible. Le cylindre est constitué d'une âme métallique recouverte d'une gaine souple de diamètre externe variable. Le courant étant constant sur la tranche d'eau, la variation de diamètre permet de simuler les effets d'un courant stratifié. Sont mesurés le mouvement du flexible en différents points et la tension dynamique en tête en fonction de la vitesse de courant et de la pré-tension en tête. Les premiers résultats mettent en évidence la complexité du comportement due au couplage entre les vibrations in-line, transverses et axiales. En particulier la déformée moyenne du flexible induite par les efforts de traînée influe fortement sur ce couplage. Par contre les modes de vibration sont nettement identifiés, ce qui permet une bonne corrélation avec les conditions d'écoulement.

- développement de nouveaux modèles pour le calcul des VIV [6]. Une première approche, dite onde, détermine les interactions entre modes dans le domaine temporel afin d'obtenir les amplitudes et les fréquences des vibrations en chaque point du flexible (information de base pour le calcul de fatigue). La deuxième approche consiste à coupler un logiciel éléments finis avec un modèle 2D Navier-Stokes prenant en compte les interférences hydrodynamiques [5]. Le couplage avec le flotteur de surface existant déjà, le modèle devient très complet. Les deux approches butent cependant sur une difficulté de taille qui est la représentation du caractère 3D de l'écoulement. La notion de longueur de corrélation est associée à un mode vibratoire et n'est actuellement accessible qu'expérimentalement. L'alternative passe par la validation de modèles Navier-Stokes 3D autour d'un cylindre déformable.

Le projet doit se poursuivre jusqu'à mi-2000. Il est mené en parallèle de campagnes d'expérimentation à grande échelle poursuivies dans le cadre JIP internationaux. Ces projets sont largement soutenus financièrement par tous les opérateurs pétroliers, les organismes de certification et les principaux contracteurs compte tenu de l'importance du problème pour les projets industriels.

\section{- 3.3 Impact des vagues extrêmes}

Un problème, mal résolu par les outils de calcul classiques, et qui fait l'objet de développements importants, est la réponse dynamique des supports flottants en mer extrême. $\mathrm{Ce}$ problème concerne d'ailleurs aussi bien la construction navale que l'offshore pétrolier. Les motivations principales concernent la prédiction :

- des mouvements de roulis et de tangage avec une prise en compte du rappel hydrostatique et des amortissements d'origine visqueuse au niveau de la carène et de ses appendices,

- des efforts d'impact des vagues sur la carène, $\mathrm{y}$ compris le slamming,

- des efforts d'impact sur les parois internes des réservoirs de liquide embarqués (sloshing),

- de l'embarquement d'eau sur le pont et de sa contribution à la stabilité du flotteur et aux efforts d'impact sur les équipements de pont.

Un bon exemple concret pour illustrer les travaux de recherche en cours est la tenue à la mer d'un navire pétrolier ancré (FPSO) sur point unique par mer sévère. Une caracté- ristique importante de ce type de navire de fort tonnage est que ses fréquences propres en tangage et pilonnement sont dans la gamme des fréquences les plus énergétiques du spectre de houle. Des analyses expérimentales et théoriques ont été menées dans le cadre du projet Clarom "Grandes surélévations de houle au voisinage d'une structure offshore flottante". Elles ont consisté à [3 ] (Fig.4) :

- réaliser des essais en bassin de houle (à l'Ecole Centrale de Nantes). Une maquette de navire a été soumise aux effets de houle de forte cambrure. Les amplitudes de la houle à l'étrave (diffraction) et les mouvements du navire ont été mesurés simultanément aux hauteurs d'eau et aux vitesses de propagation sur le pont. Les analyses ont porté principalement sur l'influence du mouvement de l'eau embarqué sur les mouvements du navire et sur les efforts d'ancrage,

- prendre en compte cette influence dans les outils de calcul de tenue à la mer classique. Le principe consiste, à l'aide d'un calcul de diffraction, à définir les conditions initiales d'envahissement puis à simuler la propagation de l'eau sur le pont à l'aide des équations de St-Venant (hypothèse de faible hauteur d'eau) simultanément au calcul des mouvements du navire, les deux étant couplés. Les résultats obtenus sont satisfaisants pour les études d'avant-projet.

- résoudre le problème complet incluant la déformée exacte de la surface libre, le franchissement, et la propagation d'eau sur le pont, les mouvements du navire. Des simulations sont réalisées avec un logiciel Navier-Stokes spécifiquement développé pour la tenue à la mer, permettant la simulation du déferlement par méthode VOF (Volume Of Fluid). Les hypothèses sont encore restrictives sur la houle incidente (houle de Stokes, onde solitaire).

Le problème du slamming est abordé parallèlement. La démarche consiste à déterminer les occurrences et les conditions d'impact à l'aide de simulation de la réponse dynamique du navire, puis à calculer les efforts locaux d'impact par une méthode prenant en compte l'intersection exacte de la carène et de la surface libre. Deux méthodes sont développées et testées : une méthode d'équation intégrale sous

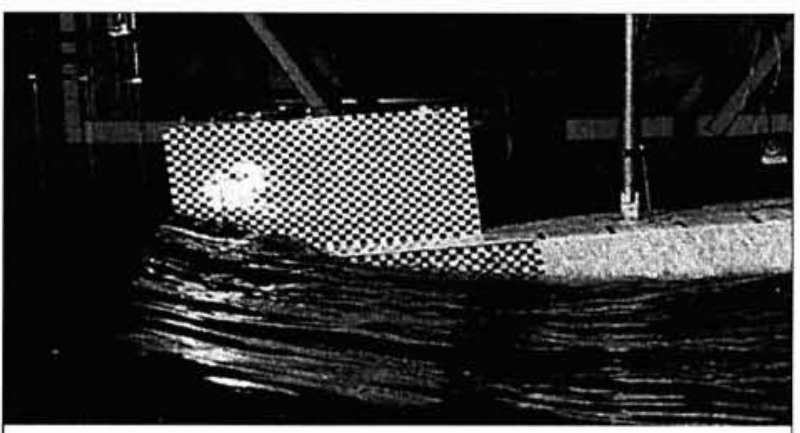

Bnvahissement d' ean d' un pont de navire

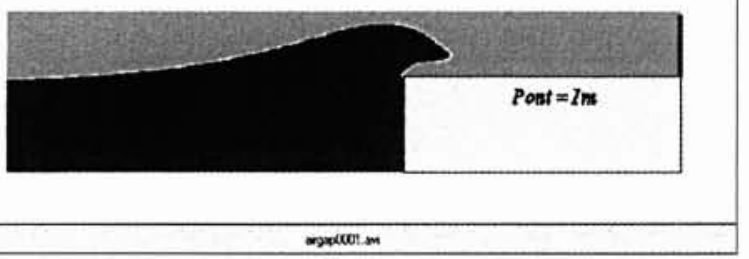

4. Exemple de modélisation expérimentale et numérique (VOF). 
l'hypothèse de fluide parfait [4], une méthode de type VOF dans un logiciel Navier-Stokes [9]. Cette dernière permettra au final d'obtenir simultanément les mouvements et les efforts d'impact.

\section{IV $\square$ CONCLUSION}

Si les outils utilisés tous les jours par l'ingénieur de projet sont très élaborés pour le calcul de la réponse dynamique d'une architecture complexe, le couplage avec les efforts hydrodynamiques reste basé sur des hypothèses restrictives (fluide parfait, linéarisation des conditions aux limites, formulations empiriques) qui nécessitent une certaine expertise de l'utilisateur et le recours à la validation expérimentale. Ces outils ont cependant le mérite d'être relativement aisés d'utilisation et de fournir des résultats assez réalistes avec un temps de réponse compatible avec l'impératif coût / délai des projets.

Les travaux de recherche menés actuellement montrent cependant que l'objectif est de disposer à moyen terme d'outils plus complets basés sur le couplage fort entre les équations de Navier-Stokes et les équations de la mécanique. Les principales difficultés à résoudre restent encore la fiabilisation des modèles de surface libre, les modèles de turbulence en écoulement instationnaire et les algorithmes de couplage de l'écoulement fluide avec la vibration des coques en grands déplacements. Dans une première étape, ces outils seront utilisés en complément des outils classiques (c'est déjà en partie le cas) compte tenu des temps de calcul nécessaires. Un effort important de validation est cependant à réaliser pour qu'ils s'imposent dans les projets industriels. Plusieurs actions sont en cours dans le cadre de projets Clarom (Roulis des barges, Cinématique des crêtes) associant les partenaires de l'offshore français. Dans le même temps la puissance CPU devrait s'être suffisamment accrue pour une utilisation réaliste en bureau d'étude.

\section{REMERCIEMENTS}

Les résultats présentés dans cette publication proviennent de deux projets Clarom (CLub pour les Actions de Recherche sur les Ouvrages en Mer) associant Ifremer, IFP, Bureau Veritas, Total, Bouygues Offshore, Doris Engineering, ETP.M, ESIM, Principia et Sirehna. Ces projets sont partiellement financés par le ministère de l'Industrie via le CEP\&M (Comité d'Etude Pétrolière et Marine).

\section{REFERENCES}

[1] Berhault C., Le Buhan P., Metals C. (1997) Hydrodynamic response of TPLs and Semis in deep water, BOSS 97 , Delft

[2] Berhault C., Boheas M.A., Morin G. (1999) Non-linear time domain analysis of complex marine operations, ISOPE' 99 . Brest

[3] Berhault C., Guérin P., Martigny D., Guéret R. (1998) Experimental and numerical investigations on the green water effects on FPSOs, ISOPE'98, Montreal

[4] Scolan Y.M., Coche E., Coudray T., Fontaine E. (1999) Etude analytique et numérique de l'impact hydrodynamique sur des carènes dissymétriques. 7èmes Journées de l'Hydrodynamique, Marseille

[5] EttenNe S., SCOLAN Y.M.. Biolley F. (1999) Modélisation numérique des écoulements de fluide visqueux autour d'un faisceau de risers. 7èmes Journées de l'Hydrodynamique, Marseille

[6] Le CunfF C., Durand A. (1999) Etude des vibrations induites par vortex : comparaison d'une approche modale et d'une approche onde. 7èmes Journées de l'Hydrodynamique, Marseille

[7] De Jouette C., Laget O., Le Gouez J.M., (1999) Vers la tenue à la me des navires par une formulation en fluide visqueux. 7èmes Journées de l'Hydrodynamique, Marseille

[8] Berhault C., Morin G., Bioliey F., Heurtier J.M. (1998), Fully coupled dynamic analysis of rigid lines and floater behaviour in deep water. ISOPE'98, Montreal

[9] Guignard S., Rey V., Marcer R. (1999), New VOF method for simulation of non-linear wave effects, ISOPE' 99 , Brest

[10] FerRANT P. (1999), Fully non-linear diffraction of regular waves by a multi-columns structure, ISOPE' 99 . Brest 